\title{
Uncovering the relationship between the structure and acid-base properties for hyperbranched polyester-polyols self-assembled on carbon surfaces is
}

\author{
Eliana D. Farias ${ }^{\text {a }}$, Valeria N. Sueldo Occello ${ }^{\text {a }}$, Mario C.G. Passeggi Jr. ${ }^{\text {b }}$, Verónica Brunetti ${ }^{\text {a,* }}$ \\ ${ }^{a}$ INFIQC, CONICET, Departamento de Fisicoquímica, Facultad de Ciencias Químicas, Universidad Nacional de Córdoba, Medina Allende y Haya de la Torre, Córdoba X500OHUA, Argentina \\ ${ }^{\text {b }}$ Laboratorio de Física de Superficies e Interfaces, IFIS Litoral (CONICET-UNL), Departamento de Física, Facultad de Ingeniería Química, Universidad Nacional del Litoral, Santa Fe, Argentina
}

\section{A R T I C L E I N F O}

\section{Article history:}

Received 28 August 2020

Received in revised form 14 October 2020

Accepted 30 October 2020

Available online xxxx

\section{Keywords:}

Hyperbranched polymer

Impedimetric titration

Surface pKa

Hydrogen-bond

\begin{abstract}
A B S T R A C T
The self-assembly of three different generations of hyperbranched polyester-polyol polymers, based on 2,2-bis (methylol)propionic acid units (H20, H30, and H40 Boltorn ${ }^{\circledR}$ ), on carbon electrodes was studied. The physicochemical properties of this family of polymers confined on a surface were explored by electrochemical impedance spectroscopy (EIS) and atomic force microscopy (AFM). Surface pKa of the hyperbranched polymer layers were determined by impedimetric titration by EIS based on the variation in the charge transfer resistance of the electroactive redox probe $\left[\mathrm{Fe}(\mathrm{CN})_{6}\right]^{4-} /\left[\mathrm{Fe}(\mathrm{CN})_{6}\right]^{3-}$ at different $\mathrm{pH}$. AFM was used to characterize the surface topography and also its viscoelastic properties by the acquisition of phase images. The relation between structure and acid-base properties can be explained in terms of a rearrangement of the layer due to the effect of intermolecular hydrogen bonds and adsorbate-substrate interactions. In this study, we report a powerful, versatile and yet simple procedure for functionalizing carbon materials surfaces without pre-treatment requirements, which could be useful to generate promising platforms for the development of sensors and drug delivery systems.
\end{abstract}

\section{Introduction}

The spontaneous adsorption of organic and/or inorganic molecules onto surfaces has been the focus of intense research due to a wide range of possible technological applications. The formation of a film over a surface based on self-assembly and physisorption is a particularly useful, simple, and economical method to modulate the surface reactivity and its properties $[1,2]$. Non-covalent interactions between adsorbates and the surface play an important role in the modification of an electrode. In particular, surface modification using dendritic molecules as adsorbates offers significant advantages regarding other polymers [3]. These macromolecules present multiple functionalities and valencies, in addition to welldefined internal cavities that favour the encapsulation of guest molecules, resulting in a synergistic interaction with the environment $[4,5]$. The resulting system usually exhibits novel features, for example, more functional groups exposed in the surface or reactive sites capable of undergoing chemical reactions [6-9]. The nature of peripheral groups also affects the interfacial behaviour of the film as a result of different types of non-covalent interactions, e.g. $\pi-\pi$ stacking, hydrogen-bond, and the hydrophobic effect. Accordingly, differences in properties, for instance viscosity, stiffness, or surface nanostructure, could be found [10-12].
Strong competitors against dendritic polymers are those named highly branched polymers or hyperbranched polymers [13]. These molecules, similarly to dendrimers exhibit exceptional properties, such as multiple terminal functional groups, specific rheological properties based on their globular structure, and well-defined internal cavities available to encapsulate guest molecules [14]. Nevertheless, the least laborious synthesis of the hyperbranched polymers [15] has motivated the design of these dendritic-like structures with numerous promising applications [16-20].

The polymers that change their properties and/or structures in response to environmental stimuli are promising candidates to modify surfaces $[21,22]$. For instance, the change of $\mathrm{pH}$ can promote the polymer shrinking/swelling and/or the adsorption/desorption of ions, nanoparticles, drugs, proteins, antigens, among others [23-26]. Consequently, these processes depend on the pKa of the functionalized surfaces, which in turn is determined by the nature of the film immobilized on the surface [22,27]. In this sense, an accurate determination of $\mathrm{pKa}$ is a significant issue to consider.

The aim of this work was to evaluate the relation between the structure and acid-base properties of hyperbranched polyester-polyols polymers selfassembled on carbon surfaces. A comparative study was carried out using three different generations of hyperbranched polymers, composed of

\footnotetext{
is This paper is dedicated to the memory of Prof. Dr Ernesto Gonzalez

* Corresponding author.

E-mail address: verobrunetti@unc.edu.ar. (V. Brunetti).
} 
2,2-bis(methylol)propionic acid units, of the Boltorn ${ }^{\circledR}$ family. The structural characterization and the pKa determination of the modified surfaces were performed by electrochemical impedance spectroscopy (EIS) and atomic force microscopy (AFM). EIS is a useful sensitive tool for studying structural changes in the macromolecular arrays and providing an initial assessment of the polymeric film permeability. The pKa values were established by impedimetric titration assessing the charge transfer behaviour of a typical redox probe at different $\mathrm{pH}$. Changes on the charge transfer resistance $\left(R_{c t}\right)$ of an external redox probe can be interpreted by the competition between intermolecular and intramolecular hydrogen bonding in the polymer layer, which control the permeation of ions (e.g., ferroferricyanide redox probes) through the film. In this work, we show that the shrinking/swelling capability of the hyperbranched polymers by an external stimulus, such as $\mathrm{pH}$, allows the environmental information to be efficiently converted into an electrochemical signal. This singularity could be applied in the development of simple and smart macromolecular devices such as sensors or drug delivery systems.

\section{Experimental section}

\subsection{Materials}

Hyperbranched polymers Boltorn ${ }^{\circledR} H 20$ (H20), Boltorn ${ }^{\circledR} H 30$ (H30), and Boltorn ${ }^{\circledR} \mathrm{H} 40$ (H40) (see structures in Fig. 1) are manufactured by Polymer Factory with a theoretical molecular weight of 1749.8, 3607.6, and $7323.3 \mathrm{~g} \mathrm{~mol}^{-1}$, respectively. Polymers solutions were prepared in anhydrous dimethyl sulfoxide (DMSO). Aqueous solutions of $0.1 \mathrm{M} \mathrm{K}_{2} \mathrm{HPO}_{4}$ / $\mathrm{KH}_{2} \mathrm{PO}_{4}(\mathrm{PBS})$ at different $\mathrm{pH}$ and $2 \mathrm{mM} \mathrm{K}_{3}\left[\mathrm{Fe}(\mathrm{CN})_{6}\right] / \mathrm{K}_{4}\left[\mathrm{Fe}(\mathrm{CN})_{6}\right]$ were prepared using ultra-purified Milli-Q water (Millipore). All solutions were prepared immediately prior to their use. All the reagents were of analytical grade and used without extra purification.

\subsection{Glassy carbon electrodes preparation}

The $3.0 \mathrm{~mm}$ diameter glassy carbon electrodes (GCE) (CH Instruments, Inc. Austin, TX) were polished with $1,0.3$, and $0.05 \mu \mathrm{m}$ alumina (Buehler), and then exhaustively rinsed with water and ethanol before its modification. After that, bare electrodes were dried with a $\mathrm{N}_{2}$ flux and incubated in a DMSO solution containing $25 \mathrm{mM}$ of $\mathrm{H} 20, \mathrm{H} 30$, or $\mathrm{H} 40$ hyperbranched polymers, at different times. After incubation, the functionalized surface was rinsed with copious volumes of ethanol and water and directly employed in the electrochemical cell.

\subsection{Electrochemical measurements}

All electrochemical measurements were performed with a $\mathrm{CH}$ Instruments Multipurpose Electrochemical Analyser at $25^{\circ} \mathrm{C}$. A single compartment three-electrode cell equipped with a glassy carbon working electrode (GCE), a platinum wire auxiliary electrode, and a $\mathrm{Ag} / \mathrm{AgCl}$ / 3.0 $\mathrm{M} \mathrm{NaCl}$ reference electrode from BAS, was used. All the potentials were referred to this electrode. Nitrogen gas was employed to deaerate aqueous solutions previous to their use. The impedance data was evaluated by non-linear least squares using the Zview ${ }^{\circledR}$ software [28]. An amplitude perturbation of $10 \mathrm{mV}$ was employed for EIS at frequencies from $100 \mathrm{kHz}$ to $0.01 \mathrm{~Hz}$.

\subsection{Atomic force microscopy (AFM)}

A commercial Nanotec Electronic SPM System with a PLL/dynamic measurement board operating in tapping mode was employed to acquire AFM images at room temperature in atmosphere conditions. The WS $\times$ M free software [29] was used to execute acquisition and image processing. Budget Sensors All-In-One-Al Multipurpose AFM Cantilevers

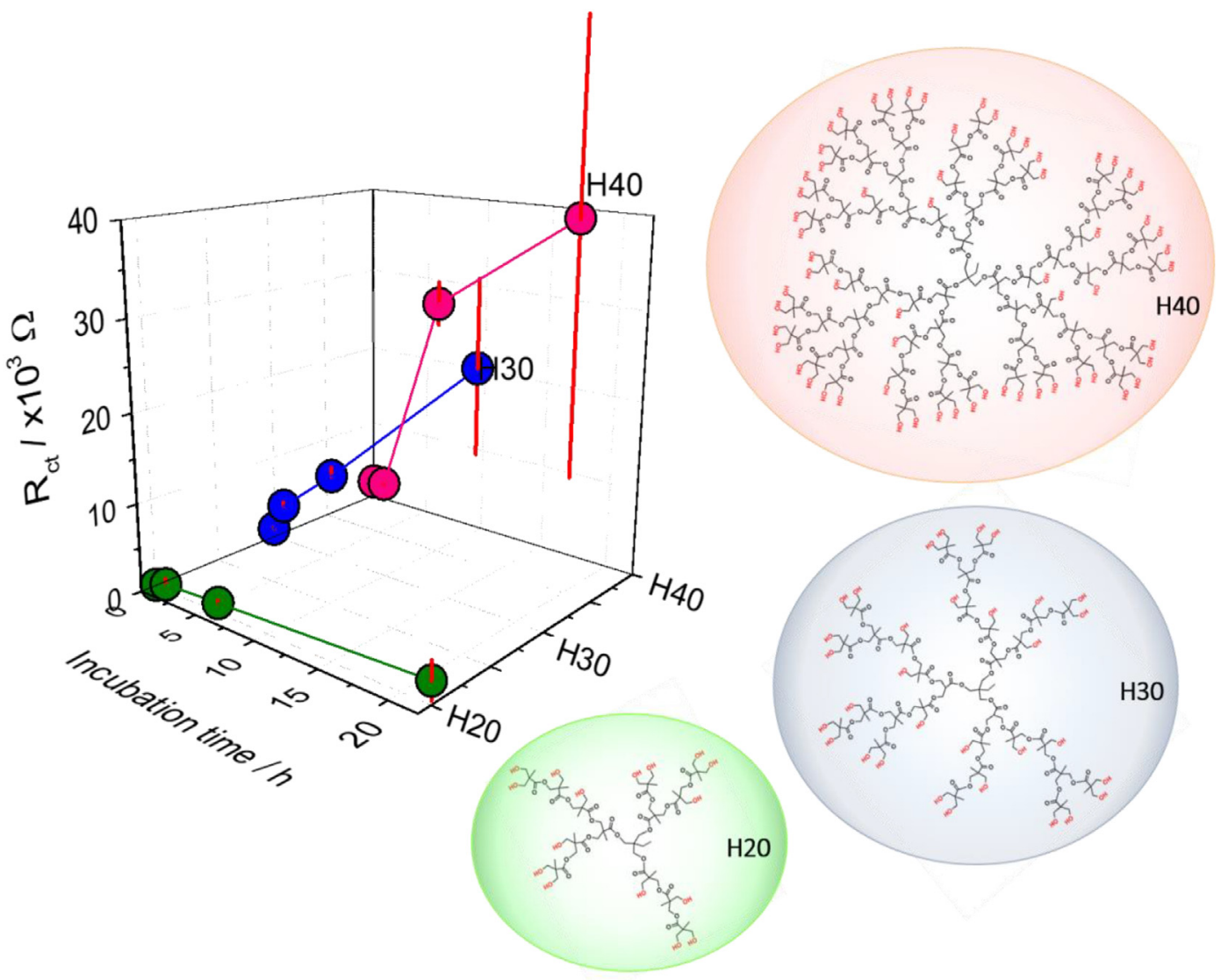

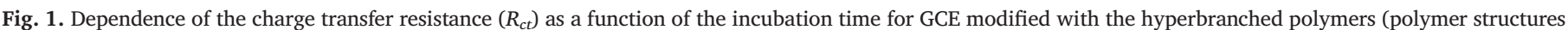
depicted in the schemes). 
(Innovative Solutions Bulgaria Ltd., Bulgaria), with a resonance frequency of $80 \mathrm{kHz}$, a nominal spring constant of $2.7 \mathrm{~N} / \mathrm{m}$, and radius of curvature minor than $10 \mathrm{~nm}$, were used for acquiring the AFM images.

Freshly cleaved highly oriented pyrolytic graphite (HOPG) surfaces were employed for AFM measurements. These surfaces were prepared by immersion in DMSO solutions containing $25 \mathrm{mM}$ of H20, H30, or H40. The surface was subsequently rinsed with abundant volumes of ethanol and water, dried under nitrogen flux and directly analysed. For statistical purposes, each sample was analysed in three different areas.

\section{Results}

\subsection{Polymer-layer structure}

The adsorption kinetics of $\mathrm{H} 20, \mathrm{H} 30$, and $\mathrm{H} 40$ were followed by EIS and correlated with the AFM information. EIS is a helpful technique to sense the interfacial properties of surface-adsorbed polymers by exploring the electron transfer behaviour of an electroactive redox probe between the electrolyte and the electrode surface. Nyquist plot of the redox probe $\left[\mathrm{Fe}(\mathrm{CN})_{6}\right]^{4-}$ / $\left[\mathrm{Fe}(\mathrm{CN})_{6}\right]^{3-}$ on bare GCE is a straight line at the entire range of frequencies with a very small semicircle at the high-frequency region indicating a small charge transfer resistance $\left(R_{c t}\right)$ and an almost fully diffusion-controlled process $[30,31]$. The impedance plots can be analysed by applying the nonlinear least squares fitting to the Randles equivalent electrical circuit, which consider the following parameters: solution resistance $\left(R_{S}\right)$, charge transfer resistance $\left(R_{c t}\right)$, double layer capacitance $\left(C_{d l}\right)$, and Warburg resistance $\left(Z_{W}\right)$ [31]. When an electrode is modified with a thin layer, the change in $R_{c t}$ of the redox probe can be used as a criterion of the film growth. In a previous work, we showed by cyclic voltammetry that the presence of a H30-layer on carbon partially inhibits the charge transfer of the redox probe at short incubation times [32]. This was determined by a shift of the anodic and cathodic peaks to more positive and negative potentials, respectively [32]. A decrease of the coulometric charge up to a totally blocked behaviour was also observed at incubation periods of ca. $24 \mathrm{~h}$ [32].

In order to gain a deeper insight into the growth of the hyperbranched polymer layers, impedance response of the redox probe $\left[\mathrm{Fe}(\mathrm{CN})_{6}\right]^{4-} /[\mathrm{Fe}$ $\left.(\mathrm{CN})_{6}\right]^{3-}$ was evaluated using carbon electrodes modified with $\mathrm{H} 20$, $\mathrm{H} 30$, and $\mathrm{H} 40$. The electrochemical measurements were performed at incubation times longer than an hour in 0.1 M PBS pH 6.8 (See S1 in the Supporting Information). The $R_{c t}$ values were determined from the extrapolation of the high frequency capacitive loop to the impedance real axis. Fig. 1 shows the dependence of $R_{c t}$ as a function of incubation time for the three highly branched polymers. At long incubation times, $R_{c t}$ exhibits a continuous increase without reaching a plateau, in good agreement with our previous results [32]. Additionally, $R_{c t}$ increases with the dendritic polymer generation (Fig. 1). The charge transport mechanism involves the diffusion of redox molecules within the film. This permeable film could present a suitable chemical environment for diffusion of the redox probe or contain pinholes where electron-transfer occurs through the movement of the electroactive ions [33]. We previously reported that the adsorption of polymers with hydroxyl-terminal groups on carbon surfaces followed a typical behaviour of an isotherm corresponding to a porous solid that forms incomplete multilayers due to hydrogen-bond forces between adsorbates [32]. Thus, the larger values of $R_{c t}$ for higher generation polymers are related to the increment of $-\mathrm{OH}$ moieties, both on internal and peripheral sites. Accordingly, hydrogen-bond interactions increase, affecting the compactness and final architecture of the organic layer.

The relationship between the polymer architecture and the film structure was explored by AFM (Fig. 2). Topographical 3D-AFM images of $\mathrm{H} 2 \mathrm{O}, \mathrm{H} 30$, and $\mathrm{H} 40$ immobilized on HOPG exhibit covered surfaces at long incubation times $(>18 \mathrm{~h}$ ). Interestingly, great differences in the roughness depending on the polymer generation were observed. The topographical data was quantitatively analysed and compared by standard roughness descriptors such as the root mean square (RMS) of the surfaces, considering height deviations. The AFM image of $\mathrm{H} 20$ presents uniformly dispersed hills with heights between 100 and $200 \mathrm{~nm}$, and a RMS value of $43 \mathrm{~nm}$. Isolated hills or cluster-like structures with heights around $90 \mathrm{~nm}$ and a slightly smooth surface formed in between by interconnected fibbers are observed for H30, with an RMS of $13 \mathrm{~nm}$. In a similar way, the AFM image of $\mathrm{H} 40$ shows a flat surface but with the presence of some globular structures formed by cluster-like agglomerates of $200 \mathrm{~nm}$ and, as consequence, the RMS is around $40 \mathrm{~nm}$. In addition, when a background area is scanned without the higher hills, a decrease in the RMS was observed (Table 1). It should also be noted that the difference between hills and valleys is several times larger than the estimated polymers diameter in good accordance to the presence of a porous multilayer (see the AFM profiles, $\mathrm{S} 2$, in the Supporting Information). Since RMS values are insufficient to describe these non-uniform surfaces, other statistical surface texture parameters, like skewness and kurtosis, are used to improve the description of the waviness at large-scale and the pitted surface at short-scale [34].

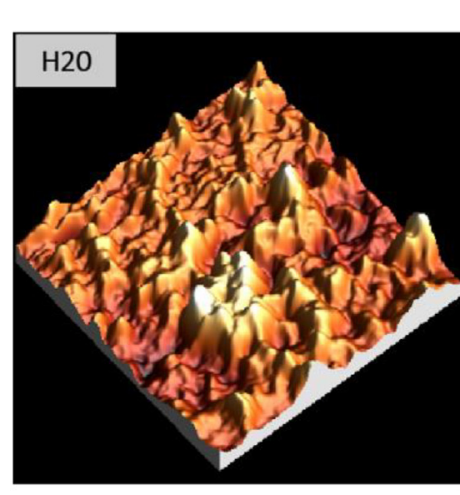

$220.00 \mathrm{~nm}$

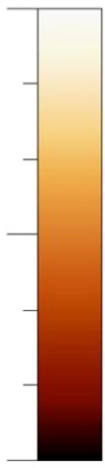

$0.00 \mathrm{~nm}$
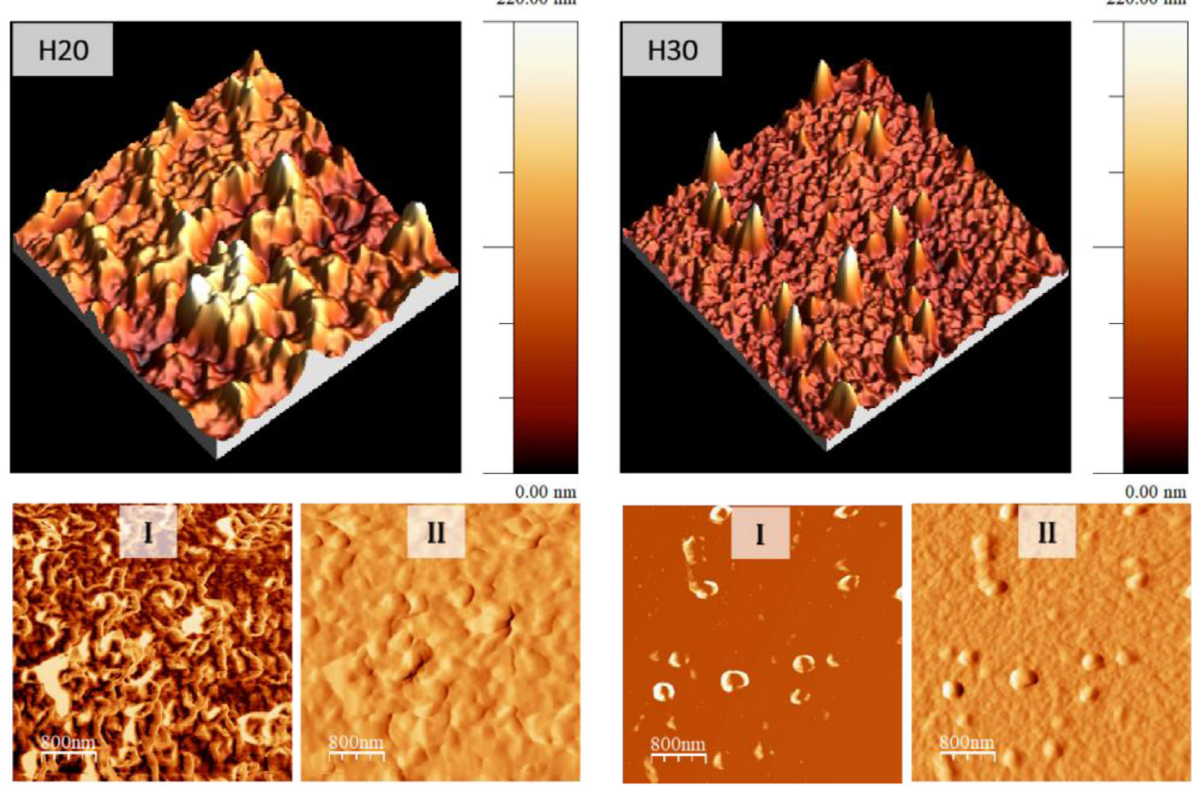

$220.00 \mathrm{~nm}$
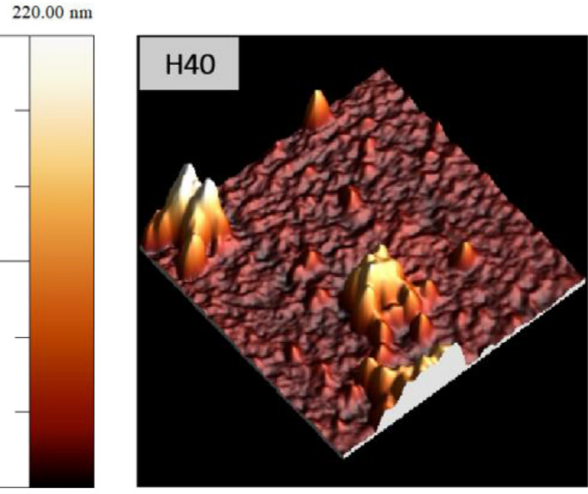

$220.00 \mathrm{~nm}$

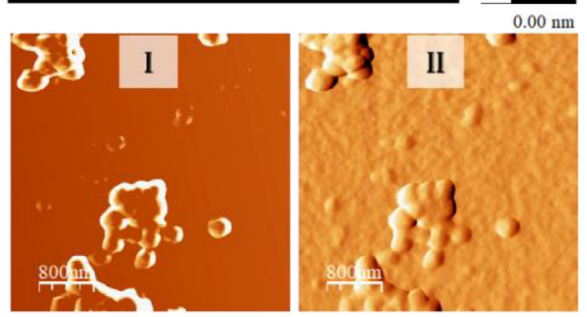

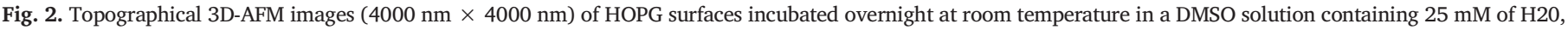
H30, or H40. Below each 3D-AFM image, their corresponding phase (I) and amplitude (II) images are shown. 
Table 1

Root mean squared roughness (RMS), skewness $\left(\mathrm{R}_{\mathrm{sk}}\right)$, and kurtosis $\left(\mathrm{R}_{\mathrm{ku}}\right)$ values of the different hyperbranched polymer layers.

\begin{tabular}{|c|c|c|c|c|c|c|}
\hline & \multicolumn{2}{|l|}{ H20/GCE } & \multicolumn{2}{|l|}{ H30/GCE } & \multicolumn{2}{|l|}{$\mathrm{H} 40 / \mathrm{GCE}$} \\
\hline & $4 \times 4 \mu \mathrm{m}^{2}$ & $0.5 \times 0.5 \mu \mathrm{m}^{2}$ (background) & $4 \times 4 \mu \mathrm{m}^{2}$ & $0.5 \times 0.5 \mu \mathrm{m}^{2}$ (background) & $4 \times 4 \mu \mathrm{m}^{2}$ & $0.5 \times 0.5 \mu \mathrm{m}^{2}$ (background) \\
\hline RMS (nm) & $(43.0 \pm 0.2)$ & $(30 \pm 6)$ & $(13.0 \pm 0.2)$ & $(6 \pm 1)$ & $(40.0 \pm 0.2)$ & $(6.0 \pm 0.5)$ \\
\hline $\mathrm{R}_{\mathrm{sk}}$ & $(0.8 \pm 0.2)$ & $(0.5 \pm 0.3)$ & $(2.4 \pm 0.2)$ & $(0.2 \pm 0.2)$ & $(2.8 \pm 0.2)$ & $(0.2 \pm 0.4)$ \\
\hline $\mathrm{R}_{\mathrm{ku}}$ & $(4.4 \pm 0.2)$ & $(2.7 \pm 0.6)$ & $(13.2 \pm 0.2)$ & $(3.5 \pm 0.8)$ & $(12.1 \pm 0.2)$ & $(4 \pm 1)$ \\
\hline
\end{tabular}

The values are reported as mean \pm standard deviation.

Table 2

Values of $k_{\text {app }}$ of the ferro/ferricyanide redox couple for the three different generations of Boltorn ${ }^{\circledR}$ polymers immobilized on GCE at different incubation times.

\begin{tabular}{|c|c|c|c|}
\hline & H20/GCE & H30/GCE & H40/GCE \\
\hline & $\operatorname{kapp}\left(\times 10^{-3} \mathrm{~cm} \mathrm{~s}^{-1}\right)$ & $\operatorname{kapp}\left(\times 10^{-3} \mathrm{~cm} \mathrm{~s}^{-1}\right)$ & $\operatorname{kapp}\left(\times 10^{-3} \mathrm{~cm} \mathrm{~s}^{-1}\right)$ \\
\hline $1 \mathrm{~h}$ & $(2.8 \pm 0.3)$ & $(0.43 \pm 0.04)$ & $(0.92 \pm 0.06)$ \\
\hline $6 \mathrm{~h}$ & $(1.7 \pm 0.4)$ & $(0.19 \pm 0.02)$ & $(0.07 \pm 0.01)$ \\
\hline$>18 \mathrm{~h}$ & $(0.77 \pm 0.08)$ & $(0.07 \pm 0.03)$ & $(0.05 \pm 0.03)$ \\
\hline
\end{tabular}

Surface skewness $\left(R_{\text {sk }}\right)$ is a measure of the statistical distribution symmetry of a surface profile [35]. A symmetric distribution should have skewness values near zero, which means that it presents evenly distributed peaks and valleys in height. Whereas surfaces with more valleys than peaks have negative asymmetry values [35]. In contrast, surfaces with more peaks than valleys are characterized by positive asymmetry values [35]. Surface kurtosis $\left(R_{\mathrm{ku}}\right)$ is a measure of the spikiness of the surface, or the distribution of spikes above and below the mean line, with a value of 3 for a normal distribution. For $R_{k u}>3$ the surface is dominated by sharp peaks, whereas if $R_{k u}<3$ the peaks are bumpy [35]. The roughness $R_{s k}$ and $R_{k u}$ parameters for $\mathrm{H} 20, \mathrm{H} 30$, and $\mathrm{H} 40$ are listed in Table 1 . The H2O layer is quite symmetric either at short or large scales with $\mathrm{R}_{\mathrm{sk}}$ value close to zero. In contrast, $\mathrm{H} 30$ and $\mathrm{H} 40$ exhibit positive values of $\mathrm{R}_{\text {sk }}$ at large-scales, indicating a major proportion of hills over the valleys. At short-scales, the roughness decreases rendering a flatter surface with $\mathrm{R}_{\mathrm{sk}}$ values close to zero. The $\mathrm{R}_{\mathrm{ku}}$ value is around 3 at short-scales for the three systems, and greater than 3 at large-scales, being notorious for $\mathrm{H} 30$ and $\mathrm{H} 40$ layers as a consequence of the presence of sharp peaks.

The phase (I) and amplitude (II) images for each polymer surface complement the roughness description (Fig. 2). In phase images, the contrast is associated with differences in the viscoelastic properties of the surface $[36,37]$. A soft cluster-like material with hard (shiny) edges and uniform characteristics across the entire surface is observed for the H2O layer. On the contrary, large and harder coalesced surfaces without contrast differences, with some cluster-shaped isolated and agglomerated structures with hard glossy edges are observed for $\mathrm{H} 30$ and $\mathrm{H} 40$ layers, respectively, all features that suggest surfaces with higher viscoelasticities compared to H20. The amplitude images show similar topographical information, revealing the map of the slope of each sample and defining the borders of the structures on the surface. Amplitude images confirm that H2O layers are formed by quasi-globular/interconnected layers while the polymers of the Pseudo Generation 3 and 4 exhibit more compact films. All these structural data point to the $\mathrm{H} 20$ layer presenting a greater number of channels than $\mathrm{H} 30$ and H40. In a previous work, we have already described the porous nature of these polymer layers based on adsorption isotherms [32]. Considering that the diffusion of redox probes takes place through the pores, these results are in good agreement with the EIS data, which show smaller charge transfer resistance values for $\mathrm{H} 20$ in comparison with H30 or H40 layers, even at long incubation times, as it can be observed in Fig. 1.


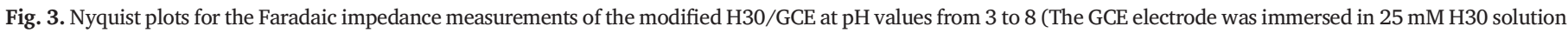

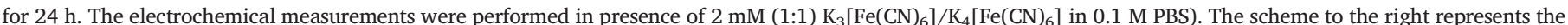
swelling of the polymer layer when $\mathrm{pH}$ decreases. 
The apparent electron transfer rate constant $\left(k_{a p p}\right)$ for modified electrodes can be calculated from EIS data using the following equation Eq 1 [38]:

$R_{c t}=\frac{R T}{n^{2} F^{2} A C^{*} k_{a p p}}$

where $R$ is the universal gas constant, $T$ is the temperature in Kelvin, $F$ is the Faraday constant, $C^{*}$ is the bulk concentration of the redox couple, $A$ is the electrode area $\left(\mathrm{cm}^{2}\right)$, and $n$ is the number of electrons involved [38].

The apparent electron transfer rate constants of the ferro/ferricyanide redox couple in aqueous electrolyte, using the electrodes modified with different polymers and incubation periods, are summarized in Table 2. In all cases, the $k_{\text {app }}$ values are at least one order of magnitude lower compared to the bare GCE electrode $\left(12.7 \times 10^{-3} \mathrm{~cm} \mathrm{~s}^{-1}\right)$. For electrodes modified with the same polymer, $k_{\text {app }}$ decreases as the incubation time increases. Additionally, for long periods of incubation, the $k_{\text {app }}$ decreases almost an order of magnitude depending on the hyperbranched polymer generation growth. The architecture of the polymer has a direct effect on the electron transfer for external redox probes. This suggests that the increment of $-\mathrm{OH}$ moieties and, in consequence, the hydrogen bondings, induces a more compact and cross-linked layer, which is reflected on the $k_{\text {app }}$ value obtained. The $k_{\text {app }}$ normally depends on various factors such as the structure and morphology of the carbon material [38]. In these systems, it could be related to the presence of ion channels/pinholes on the polymer layer, where electron-transfer occurs through the movement of the electroactive ions. These results evidence the difficulty of the heterogeneous electron transfer reaction to take place in dense packed layers of hyperbranched polymers of Pseudo Generation 3 and 4. It is important to note that these electrochemical results are in concordance with the topological parameters obtained from AFM measurements.

\subsection{Acid-base properties}

A better understanding of how the surface responds to the environmental conditions ( $\mathrm{pH}$, temperature) allows the development of surfaces with controllable properties and/or functionalities. A common method to determine acid-base properties of the electrodes modified by self-assembled monolayers (SAM) involves running cyclic voltammograms of the ferricyanide-ferrocyanide redox probe and measuring the peak current $\left(i_{p}\right)$ for this couple as a function of $\mathrm{pH}$ [39]. EIS technique is also used to determine the surface pKa by exploiting the barrier effect arising from the charged nature of SAM-pendant groups against the electrochemistry of a redox probe [40]. This last approach has been extended to polymer brushes regarding the response rate of the chemical and structural changes taking place inside the brush upon protonation and deprotonation at different $\mathrm{pH}$ values [30,41].

The impedance response of GCE electrodes modified with the hyperbranched polymers depends on $\mathrm{pH}$ (Fig. 3). The Nyquist plot shows a decrease of the ability of the film to block the diffusion of the redox probe as the $\mathrm{pH}$ diminishes. This result suggests a restructuring of the organic layer at acidic $\mathrm{pH}$, which would favour the diffusion of the redox probe towards the electrode. A possible schematic representation of the system is shown in Fig. 3. The swelling of the polymeric layer would lead to an
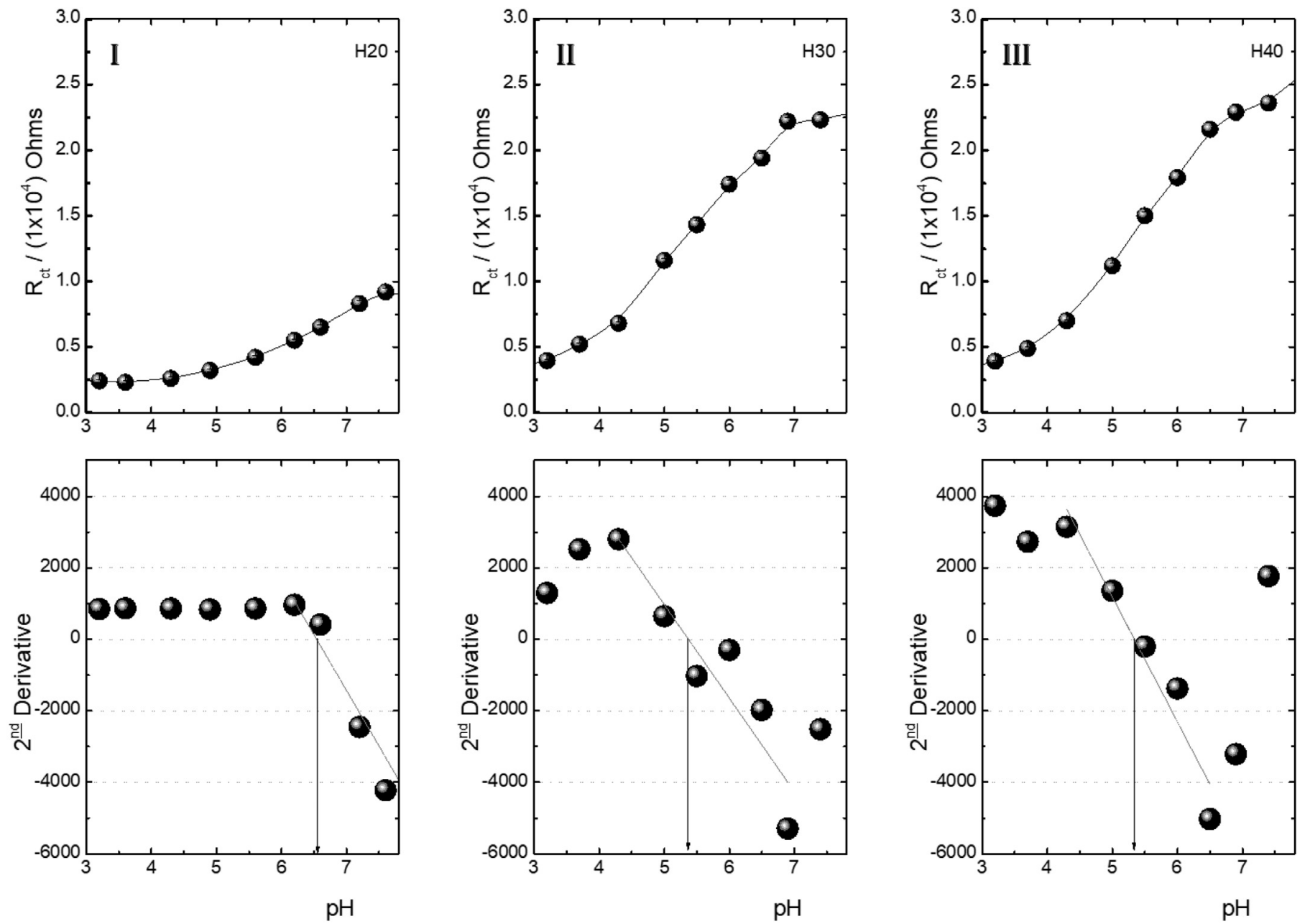

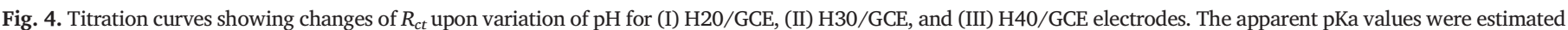
from the inflection point of the second derivative plots (shown below) of the corresponding impedimetric titration curves. 
increase of the number/width of channels that would favour the diffusion of ferro/ferricyanide species.

The impedance spectra obtained experimentally was fitted by a Randles equivalent circuit to obtain the corresponding $R_{c t}$. The $R_{c t}$ values of the modified electrodes are two orders higher than the corresponding ones of the bare electrode, confirming the presence of the polymer films. The $R_{c t}$ values were plotted as a function of the $\mathrm{pH}$ solution in order to obtain the impedimetric titration curves (Fig. 4). In all cases, the charge transfer resistance increases with an increment in $\mathrm{pH}$. Nevertheless, the changes of $R_{c t}$ are more pronounced for H30/GCE and H40/GCE, in comparison to H20/GCE. The values of the apparent surface pKa were calculated from the second derivative of these curves.

Usually, the pKa of a given compound at a solid-liquid interface is higher than its solution-phase value, with shifts of ca. 1 pKa unit or less. Despite larger shifts of surface pKa values have been reported, such as that of benzoic acid covalently attached to carbonaceous surfaces [42], the values determined for the three systems in this work are very different than the expected pKa values for primary alcohols in bulk solution ( $\mathrm{pKa}>12$ ) [43]. A plausible alternative explanation would be attributing the observed $\mathrm{pH}$ dependence to the swelling behaviour of the polymeric film. For instance, the swelling of hydrogels composed of cellulose and cellulose nanocrystals displays a dramatic $\mathrm{pH}$ response [44]. The interaction between the cellulose nanocrystals and cellulose chains is mainly due to hydrogen bonds. The swelling/deswelling is explained in terms of an increase in the ionic concentration, which in turn gives rise to an osmotic pressure. In this work, an increase of the interfacial hydrogen ion concentration within the layer structure leads to the rupture of the H-bonding network and swelling of the polymers. The interfacial charge transfer resistance for the systems observed herein would then originate from the $\mathrm{pH}$-induced swelling of the hyperbranched polymer that results in the formation of ion channels. In addition, it should be noted that although there is no significant difference between surface pKa values for H30 and H40 on GCE (pKa = 5.3), the surface pKa for H20/GCE is ca. 1.3 units higher. This suggests that lateral interactions on the carbon surface, e.g. hydrogen bonds, are responsible for the observed changes in $\mathrm{pKa}$, reflecting different ionic permeability of the nanostructured thin polymer layer. Thus, the self-assembly of highly branched polymer films on GCE allows a facile construction of nanostructured platforms in which their ionic permeability can be manipulated in response to $\mathrm{pH}$ changes.

\section{Conclusions}

In the present work, a family of three different generations of hyperbranched polyester-polyol physisorbed on glassy carbon electrodes was investigated by EIS and AFM. These self-assembled polymers form a nanostructured porous layer with multiple peripheral -OH functionalities, which give the layers a precise tuning of their ionic permeability in response to two simple control parameters: the hyperbranched polymer pseudo generation and incubation time. The electrochemistry of $\left[\mathrm{Fe}(\mathrm{CN})_{6}\right]^{4-}$ / $\left[\mathrm{Fe}(\mathrm{CN})_{6}\right]^{3-}$ redox probe was also exploited to determine the pKa of the nanostructured layers on glassy carbon surfaces. The reorganization of the polymeric film by changes in the $\mathrm{pH}$ value would result in the shrinking/ swelling of the polymers. This means that these $\mathrm{pH}$-responsive polymer interfaces are able to efficiently transduce environmental information into an electrochemical signal, and this uniqueness could be applied in the production of simple and smart macromolecular devices.

\section{Declaration of Competing Interest}

None.

\section{Acknowledgements}

The authors acknowledge financial support of ANPCyT (PICT 20172893) and SECYT-UNC (33620180100992CB). V.N.S.O. acknowledges the post-doctoral fellowship from CONICET. E.D.F., M.C.G.P., and V.B. are permanent researchers of CONICET. Finally, we all thank the Laboratory of Physics of Surfaces and Interfaces (LASUI, IFIS Litoral, CONICET$\mathrm{UNL}$ ) for the use of their SPM equipment

\section{Appendix A. Supplementary data}

Supplementary data to this article can be found online at https://doi. org/10.1016/j.jelechem.2020.114819.

\section{References}

[1] G.M. Whitesides, B. Grzybowski, Self-assembly at all scales, Science 295 (2002) 2418-2421, https://doi.org/10.1126/science.1070821.

[2] J.C. Love, L.A. Estroff, J.K. Kriebel, R.G. Nuzzo, G.M. Whitesides, Self-assembled monolayers of thiolates on metals as a form of nanotechnology, Chem. Rev. 105 (2005) 1103-1170, https://doi.org/10.1021/cr0300789.

[3] J.I. Paez, M. Martinelli, V. Brunetti, M.C. Strumia, Dendronization: a useful synthetic strategy to prepare multifunctional materials, Polymers 4 (2012) 355-395, https:// doi.org/10.3390/polym4010355.

[4] D.A. Tomalia, The dendritic state, Mater. Today 8 (2005) 34-46, https://doi.org/10. 1016/S1369-7021(05)00746-7.

[5] C. Park, J. Lee, C. Kim, Functional supramolecular assemblies derived from dendritic building blocks, Chem. Commun. 47 (2011) 12042-12056, https://doi.org/10.1039/ c1cc11531f.

[6] T. Imae, M. Ito, K. Aoi, K. Tsutsumiuchi, H. Noda, M. Okada, Formation of organized adsorption layers by amphiphilic dendrimers, Colloids Surf. A Physicochem. Eng. Asp. 175 (2000) 225-234, https://doi.org/10.1016/S0927-7757(00)00503-3.

[7] D.H. Kim, O.J. Lee, E. Barriau, X. Li, A.M. Caminade, J.P. Majoral, H. Frey, W. Knoll, Hybrid organic-inorganic nanostructures fabricated from layer-by-layer self-assembled multilayers of hyperbranched polyglycerols and phosphorus dendrimers, J. Nanosci. Nanotechnol. 6 (2006) 3871-3876, https://doi.org/10.1166/jnn.2006.669.

[8] A. Wang, J.M. Nöel, D. Zigah, C. Ornelas, C. Lagrost, D. Astruc, P. Hapiot, Electrontransfer mediation on poly-aryl dendrimer-modified electrodes, Electrochem. Commun. 11 (2009) 1703-1706, https://doi.org/10.1016/j.elecom.2009.06.026.

[9] R. Soleyman, M. Adeli, Impact of dendritic polymers on nanomaterials, Polym. Chem. 6 (2015) 10-24, https://doi.org/10.1039/c4py01208a.

[10] C. Zhang, J. Wang, Natural rubber/dendrimer modified montmorillonite nanocomposites: mechanical and flame-retardant properties, Materials 11 (2018) 41, https://doi. org/10.3390/ma11010041.

[11] B. Vivek, P. Kumar, E. Prasad, Induction and tunability of self-healing property of dendronbased hydrogel using clay nanocomposite, J. Phys. Chem. B 120 (2016) 5262-5271, https://doi.org/10.1021/acs.jpcb.6b00935.

[12] C. Zhang, J. Wang, Y. Zhao, Effect of dendrimer modified montmorillonite on structure and properties of EPDM nanocomposites, Polym. Test. 62 (2017) 41-50, https://doi. org/10.1016/j.polymertesting.2017.06.006.

[13] M. Jikey, M. Kakimoto, Hyperbranched polymers: a promising new class of materials, Prog. Polym. Sci. 26 (2001) 1233-1285, https://doi.org/10.1016/S0079-6700(01) 00018-1.

[14] E.D. Farias, M.C.G. Passeggi Jr., V. Brunetti, Películas nanoestructuradas de polímeros hiperramificados como plantillas para la formación de estructuras metálicas, Matéria 20 (2015) 772-778, https://doi.org/10.1590/S1517-707620150003.0082.

[15] Y. Zheng, S. Li, Z. Weng, C. Gao, Hyperbranched polymers: advances from synthesis to applications, Chem. Soc. Rev. 44 (2015) 4091-4130, https://doi.org/10.1039/ C4CS00528G.

[16] E.D. Farias, L.M. Bouchet, V. Brunetti, M.C. Strumia, Dendrimers and dendronized materials as nanocarriers, Nanostructures for Novel Therapy, Elsevier, Amsterdam, Netherlands 2017, pp. 429-456, https://doi.org/10.1016/B978-0-323-46142-9. 00016-5.

[17] G. Tang, X. Wang, D. Li, Y. Ma, D. Wu, Fabrication of POSS-embedded supramolecular hyperbranched polymers with multi-responsive morphology transitions, Polym. Chem. 9 (2018) 5377-5384, https://doi.org/10.1039/C8PY01271G.

[18] S.I. Bhat, Y. Ahmadi, S. Ahmad, Recent advances in structural modifications of hyperbranched polymers and their applications, Ind. Eng. Chem. Res. 57 (2018) 10754-10785, https://doi.org/10.1021/acs.iecr.8b01969.

[19] X. Liu, C. Zhu, L. Xu, Y. Dai, Ya Liu, Y. Liu, Green and facile synthesis of highly stable gold nanoparticles via hyperbranched polymer in-situ reduction and their application in $\mathrm{Ag}^{+}$detection and separation, Polymers 10 (2018) 42, https://doi.org/10.3390/ polym10010042.

[20] A. Jiménez, M.P. García-Armada, J. Losada, C. Villena, B. Alonso, C.M. Casado, Amperometric biosensors for NADH based on hyperbranched dendritic ferrocene polymers and Pt nanoparticles, Sensors Actuators B Chem. 190 (2014) 111-119, https://doi. org/10.1016/j.snb.2013.08.072.

[21] J. Khandare, M. Calderón, N.M. Dagia, R. Haag, Multifunctional dendritic polymers in nanomedicine: opportunities and challenges, Chem. Soc. Rev. 41 (2012) 2824-2848, https://doi.org/10.1039/C1CS15242D.

[22] A.M. Wagner, D.S. Spencer, N.A. Peppas, Advanced architectures in the design of responsive polymers for cancer nanomedicine, J. Appl. Polym. Sci. 135 (2018) 46154, https://doi.org/10.1002/app.46154.

[23] Y. Stetsyshyn, K. Fornal, J. Raczkowska, J. Zemla, A. Kostruba, H. Ohar, M. Ohar, V. Donchak, K. Harhay, K. Awsiuk, J. Rysz, A. Bernasik, A. Budkowski, Temperature and 
pH dual-responsive POEGMA-based coatings for protein adsorption, J. Colloid Interface Sci. 411 (2013) 247-256, https://doi.org/10.1016/j.jcis.2013.08.007.

[24] S. Ma, J. Zhou, A.R.M. Wali, Y. He, X. Xu, J.Z. Tang, Z. Gu, Self-assembly of pH-sensitive fluorinated peptide dendron functionalized dextran nanoparticles for on-demand intracellular drug delivery, J. Mater. Sci. Mater. Med. 26 (2015) 219, https://doi.org/10. 1007/s10856-015-5550-z.

[25] X. Qi, J. Qin, Y. Fan, X. Qin, Y. Jiang, Z. Wu, Carboxymethyl chitosan-modified polyamidoamine dendrimer enables progressive drug targeting of tumors via phsensitive charge inversion, J. Biomed. Nanotechnol. 12 (2016) 667-678, https://doi. org/10.1166/jbn.2016.2206.

[26] Y. Wang, D. Huang, X. Wang, F. Yang, H. Shen, D. Wu, Fabrication of zwitterionic and pH-responsive polyacetal dendrimers for anticancer drug delivery, Biomater. Sci. 7 (2019) 3238-3248, https://doi.org/10.1039/C9BM00606K.

[27] G. Kocak, C. Tuncer, V. Bütün, pH-responsive polymers, Polym. Chem. 8 (2017) 144-176, https://doi.org/10.1039/C6PY01872F.

[28] ZView® Software - Scribner Associates2020.

[29] I. Horcas, R. Fernández, J.M. Gómez-Rodríguez, J. Colchero, J. Gómez-Herrero, A.M. Baro, WS $\times$ M: a software for scanning probe microscopy and a tool for nanotechnology, Rev. Sci. Instrum. 78 (2007), 013705, https://doi.org/10.1063/1.2432410.

[30] O. Azzaroni, C. Gervasi, Characterization of responsive polymer brushes at solid/liquid interfaces by electrochemical impedance spectroscopy, Functional Polymer Films, Wiley-VCH Verlag GmbH \& Co. KGaA, Weinheim, Germany 2011, pp. 809-830, https://doi.org/10.1002/9783527638482.ch26.

[31] T.M. Nahir, Impedance Spectroscopy: Theory, Experiment, and Applications, 2nd Ed., Edited by Evgenij Barsoukov (Texas Instruments Inc.) and J. Ross Macdonald (University of North Carolina, Chapel Hill), John Wiley \& Son, ACS, 2005https://doi.org/10. 1021/ja059742o.

[32] E.D. Farias, M.C.G. Passeggi Jr., V. Brunetti, Thermal transitions in hyperbranched polyester-polyol assemblies on carbon, Eur. Polym. J. 102 (2018) 68-74, https://doi. org/10.1016/j.eurpolymj.2018.03.021.

[33] G.A. Edwards, A.J. Bergren, M.D. Porter, Chemically modified electrodes, in: C.G. Zoski (Ed.), Handbook of Electrochemistry, Elsevier, Amsterdam, Netherlands 2007, pp. 295-327, https://doi.org/10.1016/B978-044451958-0.50021-5.

[34] R. Horváth, A. Czifra, A. Drégelyi-Kiss, Effect of conventional and non-conventional tool geometries to skewness and kurtosis of surface roughness in case of fine turning of aluminium alloys with diamond tools, Int. J. Adv. Manuf. Technol. 78 (2015) 297-304, https://doi.org/10.1007/s00170-014-6642-5.
[35] B. Bhushan, Surface roughness analysis and measurement techniques, Modern Tribology Handbook: Volume One: Principles of Tribology, CRC Press 2000, pp. 49-119, https://doi.org/10.1201/9780849377877.ch2.

[36] P. Jiang, S. Xie, S. Pang, H. Gao, The combining analysis of height and phase images in tapping-mode atomic force microscopy: a new route for the characterization of thiolcoated gold nanoparticle film on solid substrate, Appl. Surf. Sci. 191 (2002) 240-246, https://doi.org/10.1016/S0169-4332(02)00184-8.

[37] R. García, R. Pérez, Dynamic atomic force microscopy methods, Surf. Sci. Rep. 47 (2002) 197-301, https://doi.org/10.1016/s0167-5729(02)00077-8.

[38] E.P. Randviir, A cross examination of electron transfer rate constants for carbon screenprinted electrodes using electrochemical impedance spectroscopy and cyclic voltammetry, Electrochim. Acta 286 (2018) 179-186, https://doi.org/10.1016/j.electacta.2018. 08.021.

[39] J. Zhao, L. Luo, X. Yang, E. Wang, S. Dong, Determination of surface pKa of SAM using an electrochemical titration method, Electroanalysis 11 (1999) 1108-1113, https://doi. org/10.1002/(SICI)1521-4109(199911)11:15<1108::AID-ELAN1108>3.0.CO;2-Z.

[40] A.R. Puente-Santiago, G. Sánchez-Obrero, T. Pineda, M. Blázquez, R. Madueño, Influence of patterning in the acid-base interfacial properties of homogeneously mixed CH3- and COOH-terminated self-assembled monolayers, J. Phys. Chem. C 122 (2018) 2854-2865, https://doi.org/10.1021/acs.jpcc.7b11878.

[41] M. Motornov, R. Sheparovych, E. Katz, S. Minko, Chemical gating with nanostructured responsive polymer brushes: mixed brush versus homopolymer brush, ACS Nano 2 (2008) 41-52, https://doi.org/10.1021/nn700214f.

[42] P. Abiman, A. Crossley, G.G. Wildgoose, J.H. Jones, R.G. Compton, Investigating the thermodynamic causes behind the anomalously large shifts in pKa values of benzoic acid-modified graphite and glassy carbon surfaces, Langmuir 23 (2007) 7847-7852, https://doi.org/10.1021/la7005277.

[43] B. Thapa, H.B. Schlegel, Improved pKa prediction of substituted alcohols, phenols, and hydroperoxides in aqueous medium using density functional theory and a clustercontinuum solvation model, J. Phys. Chem. A 121 (2017) 4698-4706, https://doi. org/10.1021/acs.jpca.7b03907.

[44] X. Gao, K.K. Sadasivuni, H.C. Kim, S.K. Min, J. Kim, Designing pH-responsive and dielectric hydrogels from cellulose nanocrystals, J. Chem. Sci. 127 (2015) 1119-1125, https://doi.org/10.1007/s12039-015-0873-3. 